\title{
CFD Analysis of 2D Unsteady Flow Past a Square Cylinder at Low Reynolds Numbers
}

\author{
Zhenquan $\mathrm{Li}^{1, *}$, and Robert Wood ${ }^{1}$ \\ ${ }^{1}$ School of Computing and Mathematics, Charles Sturt University, NSW 2640, Australia
}

\begin{abstract}
A study of the behaviour of flow past a square cylinder for Reynolds numbers 10 and 20 is presented. Open source software Navier2d in Matlab is used in this study. The investigation starts from a uniform initial mesh and then refine the initial mesh using a mesh refinement method which was proposed based on both qualitative theory of differential equations and the finite volume method implemented in Navier2d. The horizontal and vertical velocity component profiles and pressures are shown on the once refined meshes. The comparisons between the profiles and pressures are conducted to show the variations from Reynolds number 10 to 20 . The twice refined meshes are also presented and these refined meshes provide the information where the behaviour of flow is complex.
\end{abstract}

\section{Introduction}

The study on wakes of flow around a bluff body has various applications in practice such as aerodynamics, wind engineering, and electronics cooling. The flow around a square cylinder has been investigated extensively using different methods in literature [e.g. 1, 2].

We use a different computational technique to analyse the flow past a square cylinder. A 2D adaptive mesh refinement method is proposed based on the qualitative theory of differential equations and utilise an existing numerical method [4]. The accuracy of the method is verified by analytical and CFD velocity fields considering the location of centres of vortices and/or separating curves of different vortex regions $[5,6,8,9]$. The reliability of the method is considered and the positive outcome had been achieved [7]. The cost (computational complexity) is investigated using liddriven cavity flow and a positive outcome again is achieved [10]. We use Navier2d as the numerical method. Navier2d is a vertex centred Finite Volume (FV) code that uses the median dual mesh for the Control Volumes (CV's) about each vertex. One of the advantages of the adaptive mesh refinement method is that a mesh is refined based on the information of the calculated velocity field on the mesh so the refined mesh shows where the velocity is more complex which is presented by more nodes in the refined mesh.

In this paper, we apply the adaptive mesh refinement once to the initial mesh. We show the velocity fields and pressures calculated on the once refined mesh, and the twice refined meshes that provide the information in the areas that contains centres of vortices and separation curves of vortex regions that are in the domain of interest [9].

\section{The governing equations}

In this paper, we use Navier-Stokes equations for unsteady flow of an incompressible fluid of density as governing equations

$$
\begin{aligned}
& \frac{\partial \boldsymbol{u}}{\partial t}+\boldsymbol{u} \cdot \nabla \boldsymbol{u}=-\nabla p+\frac{1}{R e} \nabla^{2} \boldsymbol{u} \\
& \nabla \cdot \boldsymbol{u}=0
\end{aligned}
$$

Where Re is the Reynolds number. The domain is shown in Fig. 1.

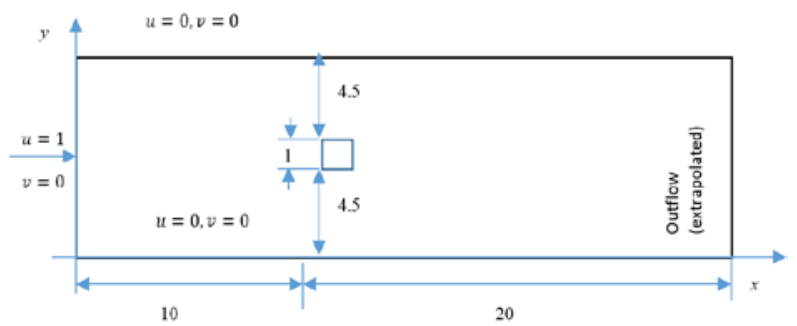

Fig. 1. Computational domain.

The domain in this study is a rectangle with the length 30 units and width 10 units. The unit square cylinder is placed inside of the rectangle with the centre located at $(10.5,5)$. The flow is from left to right. The origin of the xy coordinate system is located at the lower left-hand corner of the rectangle and the $\mathrm{x}$ axis is in the downstream direction.

The boundary condition at the inlet is $\mathrm{u}=1$ and $\mathrm{v}=0$. On the top and bottom length of the rectangle and the boundary of the square cylinder, $\mathrm{u}=\mathrm{v}=0$. The boundary

\footnotetext{
* Corresponding author: jali@csu.edu.au
} 
condition at the outlet is the outflow extrapolate chosen in Navier2d.

\section{Numerical results}

We use uniform initial mesh with size $20 \times 60$. The initial mesh is refined based on the CFD velocity field calculated on the initial mesh. We then calculate the CFD velocity field on the once refined mesh. From the once refined mesh, we obtain the information about where the linearly interpolated velocity field does not follow the equivalent continuity equation [4]. The twice refined meshes are also shown. We consider the cases for Reynolds number $\mathrm{Re}=10$ and 20. Fig. 2 shows the initial mesh.

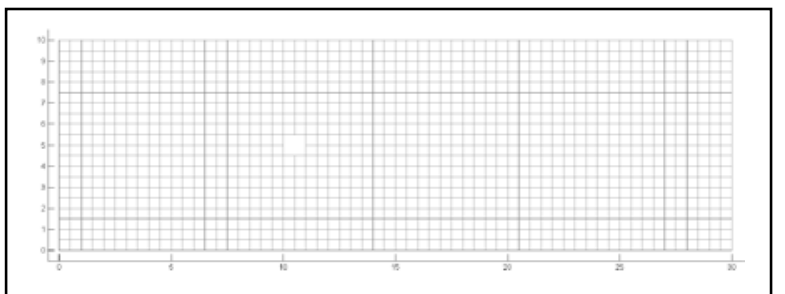

Fig. 2. Initial mesh used in study.

Navier2d requires the triangular mesh in the calculations. Fig.3 shows the triangular mesh generated from the initial mesh.

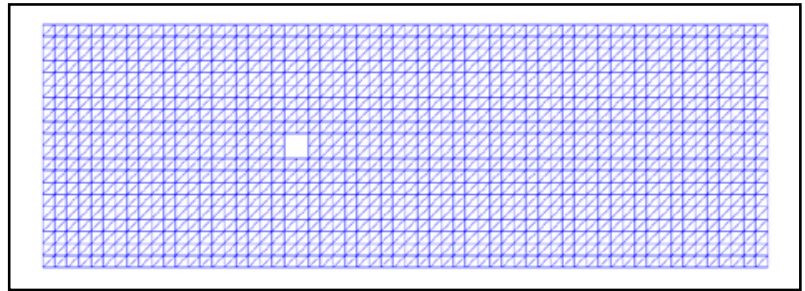

Fig. 3 Triangular mesh generated from the initial mesh.

Even though we start from the same initial mesh, the once and twice refined meshes are different due to different CFD velocity fields. The residuals in this study are less than $10^{-10}$. The top figure shows the case $R e=10$.

\subsection{Horizontal velocity comparison}

The following figures present the differences between the $u$ velocity component for the two cases $R e=10$ and 20.

From the two figures in Fig. 4, the wake of $R e=10$ is longer than that of $\mathrm{Re}=20$ and the width of the wake of $\mathrm{Re}=10$ is wider than that of $\mathrm{Re}=20$. The flow gets more complex around the cylinder for $\mathrm{Re}=20$ than that for $\mathrm{Re}=10$ from both the once refined mesh and the profiles.

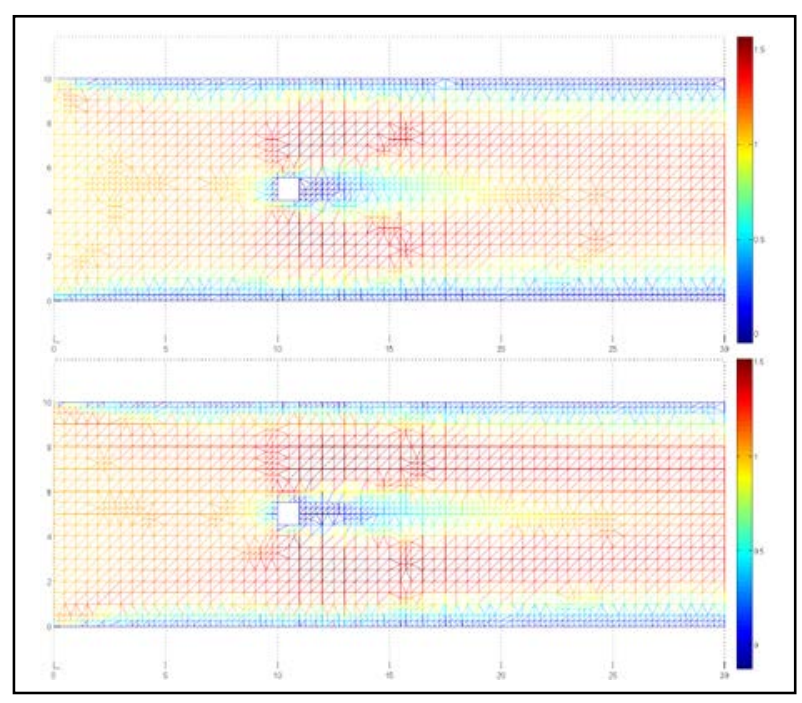

Fig. 4. The profiles of $\mathrm{u}$ components.

\subsection{Vertical velocity comparison}

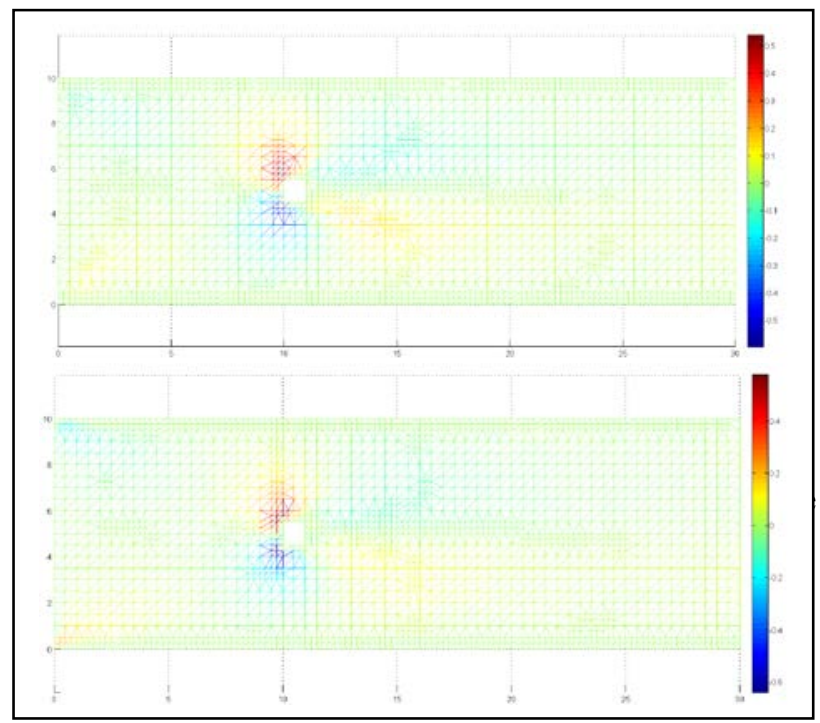

Fig. 5. The profiles of $\mathrm{v}$ components.

Fig. 5 shows the v profiles for both cases, in each case there are less differences than displayed in the $u$ profile.

\subsection{Pressure comparison}

The comparison of pressures is shown in Fig. 6. There are large differences between the pressures. The pressure before the cylinder is larger for $\mathrm{Re}=10$ than that of the case $R e=20$ since the flow is faster in the latter case. 


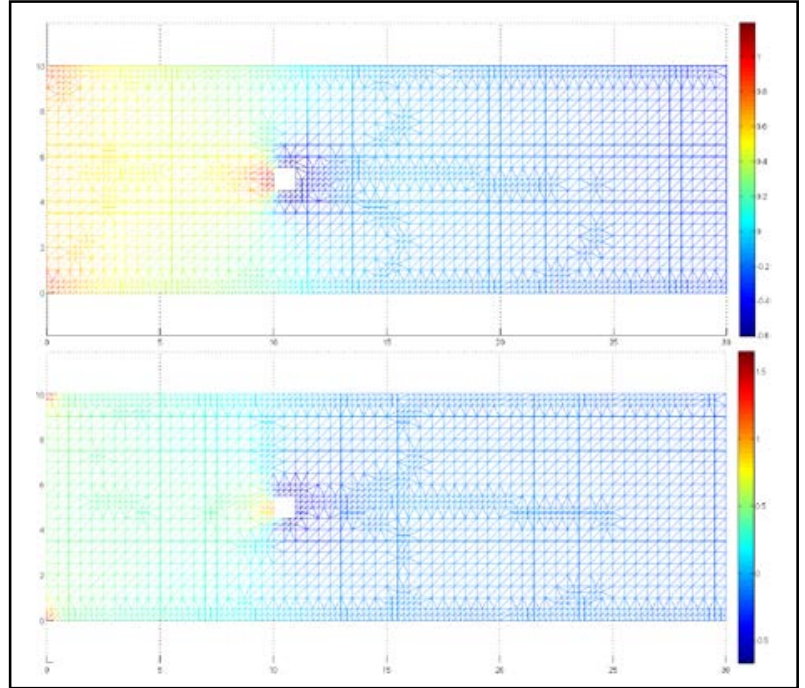

Fig. 6. Pressure profiles.

\subsection{Twice refined meshes}

The twice refined meshes are shown in Fig. 7 for both cases. In the region before the cylinder, the case $\mathrm{Re}=10$ has a higher pressure so this area requires more fine grids as indicated in the top graph of Fig. 7. After the cylinder, the case $\mathrm{Re}=20$ has a longer tail and the velocity field is sensitive in the vicinity of the tail. This is also indicated in the bottom graph of Fig. 7.

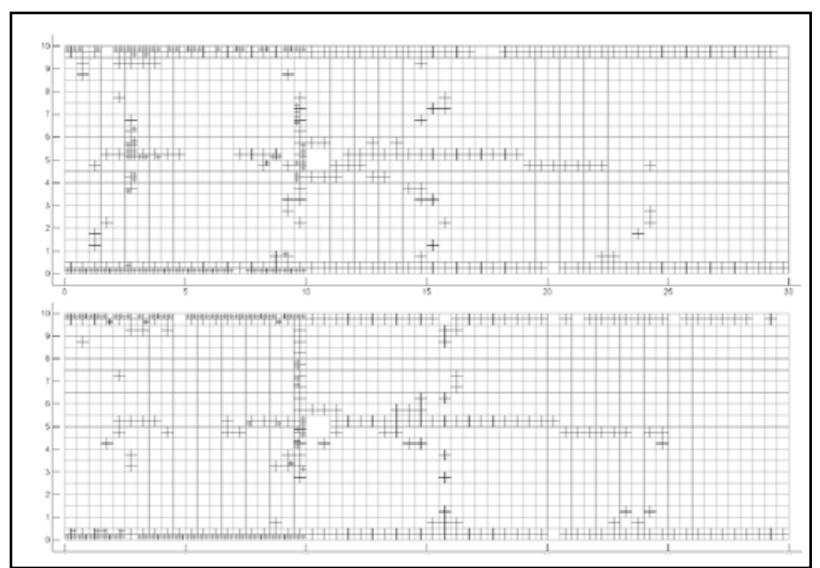

Fig. 7. Twice refined meshes.

\subsection{Velocity fields}

Fig. 8 shows the velocity fields calculated from Navier2d on the once refined mesh for both cases. For the case $R e=10$ in the top figure, the wake is shorter when compared with that of the bottom figure which is the case $R e=20$. There are larger differences between the two velocity fields after the cylinder. We also see the errors in these two figures. The domain is symmetrical, the boundary and initial conditions are the same for the two regions separated by $y=5$. However, the velocity fields are not symmetrical. Comparing the figures in Fig.
7 and Fig. 8, we conclude that the twice refined meshes are totally dependent of the numerical velocity fields calculated which is the same as we found before.

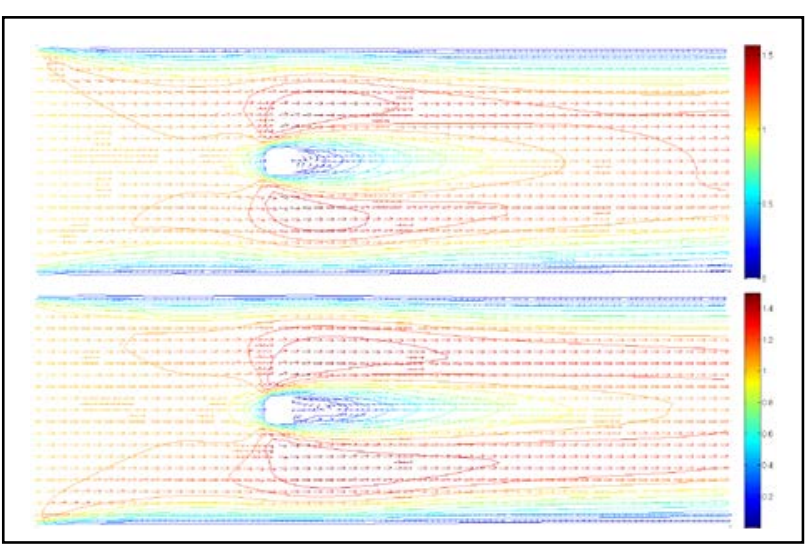

Fig. 8. Velocity fields.

\section{Discussion}

Even though the results from the uniform initial are not presented in this paper, our previous research has shown that the refined meshes generate more accurate results.

We have shown the simple cases in this paper. More complex cases are being investigated. The boundary conditions used in this paper are different from the boundary conditions used in $[1,2]$. There are practical problems following the boundary conditions described in Section 2.

In the future, we will consider improving the accuracy of the numerical velocity fields in two ways. The first way is to choose finer initial mesh and the second is more refinements performed on the initial mesh.

\section{References}

1. S. Sen, S. Mittal, G. Biswas, Int. J. Numer. Meth. Fluids, 67, 1160-1174(2011). DOI : 10.1002/fld. 2416

2. B. Gera, P.K. Sharma, P.K. Singh, Int. J. Appl. Eng. Res., 1, 602-610(2010).

http://ipublishing.co.in/jarvol1 no12010/EIJAER202 8.pdf

3. D. Engwirda, Navier-Stokes solver (Navier2d). MATLAB Central File Exchange, 2006.

4. Z. Li, J. Flow Visual. Image Process., 15, (2008). DOI: 10.1615/JFlowVisImageProc.v15.11.20

5. Z. Li, J. Flow Visual. Image Process., 13, 1(2006). DOI: 10.1615/JFlowVisImageProc.v13.11.10

6. Z. Li, J. Math. Chem., 52, 4(2014). DOI : 10.1007/s10910-014-0334-0

7. R. Lal, Z. Li, J. Math. Chem., 53, 3(2015). DOI: 10.1007/s10910-014-0461-7

8. Z. Li, J. Comput. Appl. Math., 275, (2015). DOI: 10.1016/j.cam.2014.07.025

9. Z. Li, R. Wood, J. Comput. Appl. Math., 318, (2017). DOI : 10.1016/j.cam.2016.09.022

10.Z. Li, Comput. Therm. Sci., to appear. 